\title{
Pulmonary Hypertension and the Heart: a Complex Interaction
}

\author{
Robert Naeije, MD, PhD
}

Department of Cardiology, Erasme University Hospital, ULB, Brussels, Belgium

\section{ABSTRACT}

Pulmonary hypertension primarily affects the heart by increasing right ventricular (RV) afterload. The initial response of the RV is an increased contractility soon followed by hypertrophy, with little or no increase in dimensions. When this "homeometric" adaptation fails, in case of rapid and/or severe increase in pulmonary artery pressure, the RV also uses a "heterometric" adaptation at the price of increased dimensions, higher filling pressures, systemic congestion and decreased exercise capacity. Dilatation of the RV impairs left ventricular (LV) filling. This diastolic interaction eventually alters systolic interaction, or contribution of LV contraction to the strength of RV contraction, which further deteriorates RV function. Too high or low lung volumes may increase pulmonary vascular resistance. Excessive decrease in pleural pressures be a cause of LV failure and worsening of pulmonary hypertension by upstream transmission of filling pressures. The complexity of heart-lung interactions in pulmonary hypertension needs understanding to optimize treatment strategies. (BRN Rev. 2017;3:136-48)

Corresponding author: Robert Naeije, rnaeije@ulb.ac.be

Key words: Functional residual capacity. Heart-lung interactions. Pleural pressure. Pulmonary hypertension. Right ventricle. Ventricular interdependence. 


\section{INTRODUCTION}

Pulmonary hypertension $(\mathrm{PH})$, defined by a mean pulmonary artery pressure (mPAP) $\geq 25 \mathrm{mmHg}$ is a common complication of cardiac and respiratory diseases, but may sometimes present as an intrinsic pulmonary vascular disease without identifiable aetiology called pulmonary arterial hypertension $(\mathrm{PAH})^{1}$. Symptomatology, functional state, exercise capacity and outcome in $\mathrm{PH}$ are essentially to be accounted for by an altered right ventricular (RV) function adaptation to afterload ${ }^{2}$. This review will focus on the mechanisms of adaptation and failure of RV failure in $\mathrm{PH}$, with consideration of RV-arterial coupling, ventricular interdependence and heart-lung interactions.

\section{RIGHT VENTRICULAR FAILURE}

The normal RV is thin-walled and crescentshape flow generator, which is vulnerable to any acute rise in wall stress ${ }^{3}$. A brisk increase in pulmonary vascular resistance (PVR) induces acute dilatation and pump failure of the RV. However, a gradual increase in PVR allows for RV adaptation and remodelling. Beat-to-beat changes in preload or afterload are accompanied by a heterometric dimension adaptation following Starling's law of the heart ${ }^{4}$. Sustained changes in load are associated with a homeometric contractility adaptation following Anrep's law of the heart after the initial observation by Gleb von Anrep ${ }^{5}$ in 1912 of rapid increase in LV contractility in response to an aortic constriction. Failure of homeometric adaptation results in heterometric adaptation to maintain stroke volume (SV) but at the price of increased RV end-systolic volume (ESV) and end-diastolic volume (EDV) ${ }^{2,6}$.
The so-called "laws of the heart" equally apply to the RV and to the LV, but in different proportions ${ }^{6}$.

It is therefore possible to define RV failure as a dyspnoea-fatigue syndrome with eventual systemic venous congestion, caused by the inability of the RV to maintain flow output in response to metabolic demand without heterometric adaptation resulting in increased filling pressures and volumes? ${ }^{2}$.

\section{RIGHT VENTRICULAR AFTERLOAD}

When PVR is normal, around 1 Wood unit (WU) or less, the RV functions as a low pressure volume generator with minimal work at rest or at moderate levels of exercise ${ }^{3,6}$. A good quality of life is even possible without a RV, as shown by near-normal life expectancy with only mild to moderate exercise intolerance after a "Fontan operation", or cavo-pulmonary anastomosis used as palliative intervention in infants with some congenital cardiac abnormalities?. However, as soon as PVR starts to increase, the pump function of the RV is needed to accommodate systemic venous return and preserve LV pre-loading. Thus, afterload matters considerably to the RV.

There are several equally valid estimations of $\mathrm{RV}$ afterload ${ }^{8}$. The first is maximum wall tension, but which is difficult to measure because of the irregular shape of the RV and regional inhomogeneous of contraction. The second is hydraulic power $\left(\mathrm{W}_{\mathrm{TOT}}\right)$, calculated from the integration of pressure and flow waves, thus encompassing oscillatory and steady-flow components $\left(\mathrm{W}_{\mathrm{OSC}}\right.$ and $\left.\mathrm{W}_{\mathrm{ST}}\right)$. The third is arterial elastance (Ea), or end-systolic 
pressure, ESP divided by SV measured on a $\mathrm{RV}$ pressure-volume loop. Ea corresponds to afterload perceived by the RV.

Because of the near-constancy of the time constant of the pulmonary circulation, or pulmonary arterial compliance $x$ PVR, around 0.4 to 0.8 seconds, $\mathrm{W}_{\text {OSC }}$ is stable at $23 \%$ of $\mathrm{W}_{\text {TOT'}}$ and is estimated by 1.3 times mean power $\mathrm{W}_{\mathrm{ST}}{ }^{9}$ :

$$
\mathrm{W}_{\mathrm{TOT}}=1.23 \times \mathrm{W}_{\mathrm{ST}}=1.23 \times \mathrm{SV} \times \mathrm{PAP}
$$

As RV end-systolic pressure (ESP) is approximated by mPAP, Ea is estimated by a ratio of mPAP to SV:

$$
\mathrm{Ea}=\mathrm{mPAP} / \mathrm{SV}
$$

or PVR divided by heart rate.

Practically, Ea is most relevant as a single number definition of afterload the RV.

\section{RIGHT VENTRICULAR SYSTOLIC FUNCTION}

The homeometric adaptation relies on increased contractility. Myocardial fibre contractility is defined by an active tension-length relationship. In vivo, ventricular contractility is defined by a maximal elastance (Emax), or the maximum slope of a pressure-volume (PV) relationship measured continuously during the cardiac cycle - or PV loop $2,6,8$.

Emax of the LV coincides with end-systole, and is thus equal to the ratio between ESP and ESV defining an end-systolic elastance (Ees). Left ventricular Ees is clearly identifiable at the upper left corner of a square-shaped PV loop ${ }^{10}$.
Because of naturally low pulmonary vascular impedance, the normal RV PV loop has an oval rather than square shape and Emax occurs before the end of systole. However, a satisfactory definition of Emax can be obtained by a family of PV loops at decreasing venous return (as generated by progressive inferior vena cava balloon occlusion) ${ }^{11}$.

Increasing impedance to pulmonary blood flow in PH changes the shape of the RV PV loop, so that Emax becomes closer to peak systolic pressure and the slope of end-systolic $\mathrm{P}-\mathrm{V}$ relationship increases, indicating increased contractility ${ }^{12}$.

Even though there has been recent progress in three-dimensional echocardiography and magnetic resonance imaging (MRI), instantaneous volume measurements of the RV are not yet possible at the bedside. Manipulations of venous return through insertion of a vena cava balloon catheter to generate families of PV loops add to the invasiveness of right heart catheterization, and are ethically problematic. A Valsalva manoeuvre to generate a family of RV PV loops at decreased venous return was recently introduced and validated ${ }^{13}$. The practicality and clinical relevance of this approach will require confirmation.

Since Emax is insensitive to immediate changes in preload or afterload, single beat methods have been developed, initially for the $\mathrm{LV}^{14}$ and subsequently adapted to the $\mathrm{RV}^{15}$. The single beat method relies on a maximum pressure Pmax calculation from a nonlinear extrapolation of the early and late portions of a RV pressure curve, an integration of pulmonary flow and synchronization of the signals. Emax is estimated from the slope of a straight 


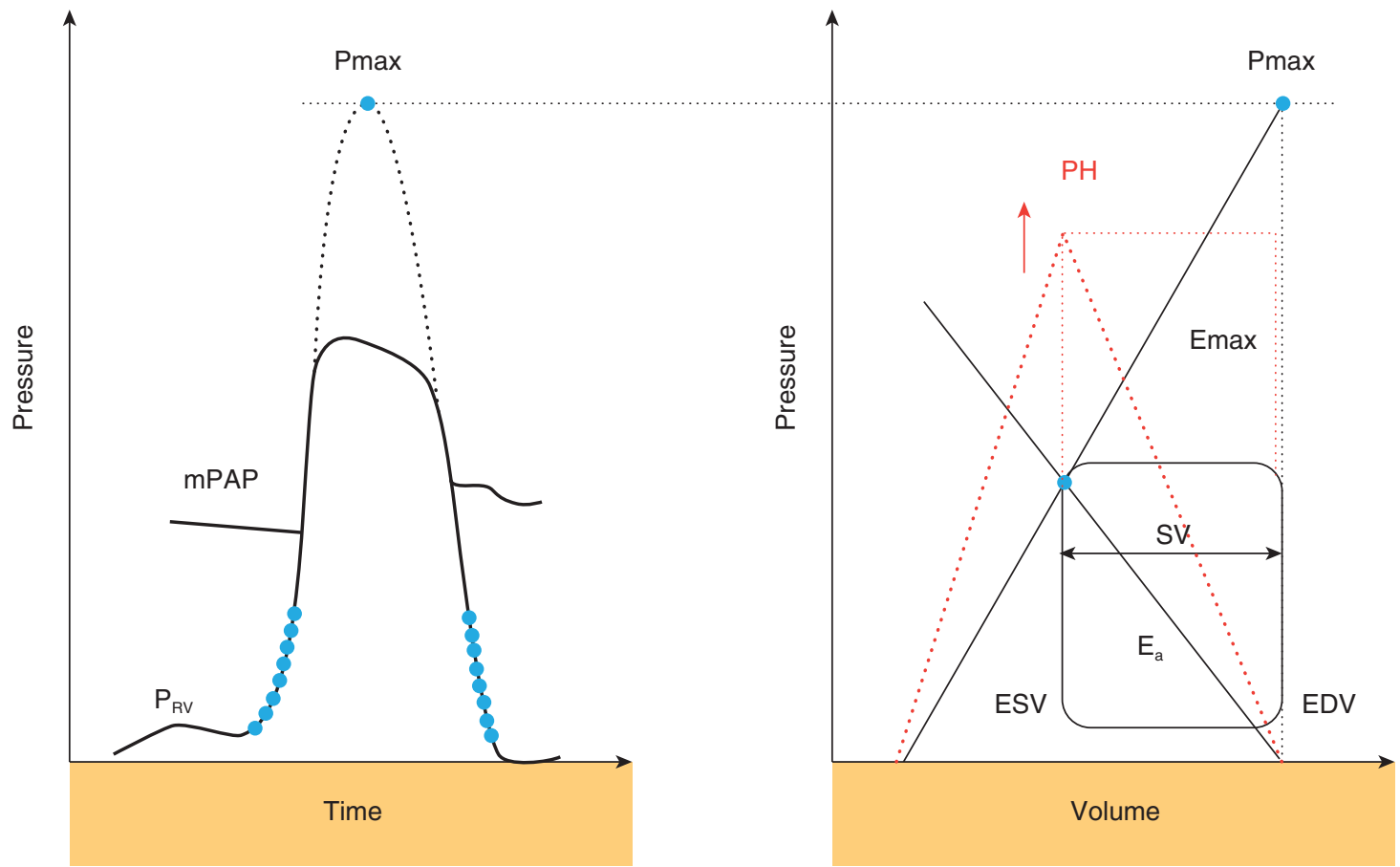

Figure 1. Simplified pressure method to calculate end-systolic elastance (Ees) and arterial elastance (Ea) based on a maximum pressure (Pmax) derived from nonlinear extrapolation of early and late portions of a right ventricular pressure curve (PRV), a measure of stroke volume (SV) and assumption that mean pulmonary artery pressure (mPAP) is a reasonable approximation of end-systolic pressure. The effects of an increase in mPAP (PH) with preserved RV-arterial coupling and no change in RV volumes is shown in red. EDV: end-diastolic volume; Emax: maximum, or end-systolic elastance; ESV: end-systolic volume; PH: pulmonary hypertension.

line tangent from Pmax to the upper left portion of the PV curve. This is illustrated in figure 1.

A Pmax corresponds to the pressure the RV would generate during a non-ejecting beat at EDV. An excellent agreement between directly measured Pmax by clamping the main pulmonary artery for one beat and calculated Pmax has been demonstrated in a large animal experimental preparation with no $\mathrm{PH}$ or a mild increase in PAP induced by low oxygen breathing ${ }^{15}$. Whether the calculated Pmax is equally valid in patients with severe $\mathrm{PH}$ has not been yet established with certainty.
Measurements of RV Emax with conductance catheter technology and inferior vena cava balloon obstruction have been reported in normal subjects ${ }^{16}$. A limited number of Emax determinations have been reported in patients with severe $\mathrm{PH}$, either PAH or thrombo-embolic $(\mathrm{CTEPH})^{13,17-20}$ and in one patient with a systemic RV on congenitally corrected transposition of the great arteries ${ }^{21}$. The methods varied, with single or multiple beat approaches, fluid-filled or micro-manometer-tipped catheters to measure pressures, and conductance technology or magnetic resonance imaging to measure volumes. Emax consistently increased in response to increased Ea. 


\section{COUPLING OF RV SYSTOLIC FUNCTION TO AFTERLOAD}

Ventricular adaptation to increased preloading or afterloading is initially heterometric, so that ESV and EDV are increased with preserved or increased SV but decreased ejection fraction (EF). This was established by Starling in heart-lung preparations. However, after 20 to 30 seconds, ventricular contractility increases returning volumes to pre-intervention state. This "homeometric adaptation" results in increased or preserved SV and unchanged or decreased ESV and unchanged EDV ${ }^{22}$. It is thus important to correct measurements of contractility for increased afterload.

Contractility corrected for afterload is best defined by a ratio of Emax to Ea. Experimental work and mathematical modelling have allowed the definition of an optimal mechanical coupling of Emax to Ea equal to one. However, the optimal condition for energy transfer from the ventricle to the arterial system occurs at a Emax/Ea ratio of 1.5-22,6,8.

Measurements of the Emax/Ea ratio have been reported in various models of $\mathrm{PH}^{6}$. RV-coupling was preserved with increased Emax to match Ea in models of hypoxic pulmonary vasoconstriction, pulmonary embolism, pulmonary artery banding, early endotoxic shock and short term (3 months) aorta-pulmonary shunting in piglets. This is schematically illustrated in figure 1 . However, RV-arterial coupling was deteriorated with a decrease in the Emax/Ea ratio in late endotoxic shock, monocrotaline-induced $\mathrm{PH}$, long-term (6 months) aorta-pulmonary shunting in piglets and mild $\mathrm{PH}$ in overpacing-induced heart failure. Deterioration of $\mathrm{RV}$-arterial coupling was associated with increased EDV. There is thus compelling experimental evidence of predominant RV systolic function adaptation to increased afterload in $\mathrm{PH}$, but with early RV-arterial uncoupling and increased RV volumes in the context of inflammation (endotoxemia, monocrotaline), long-term increase in PVR (systemic-to-pulmonary shunting), or heart failure.

Measurements of both Emax and Ea have been reported in a limited number of patients with $\mathrm{PAH}$ or $\mathrm{CTEPH}^{13,17-20}$. In the presence of increased Ea, Emax was always increased but with either preserved or decreased Emax/Ea. A decreased Emax/Ea was more constantly observed in patients with PAH associated with systemic sclerosis (SSc) ${ }^{13,20}$. Exercise decreased Emax/Ea even in those patients with a normal Emax/Ea at rest, in contrast to preserved Emax/Ea during exercise in controls without $\mathrm{PH}^{19,20}$. A decrease in Emax/Ea during exercise was associated with a decreased EDV ${ }^{20}$ confirming current notion that a decrease in Emax/Ea at some point in the course of severe $\mathrm{PH}$ as associated with increased right heart chamber dimensions, increased central venous pressures, systemic congestion, and decreased life expectancy ${ }^{2,23}$.

The ventricular homeometric adaptation to increased afterload is eventually but variably strengthened by a hypertrophic response. The increase in (right) ventricular wall thickness (h) in (pulmonary) hypertension is adaptative as, according to Laplace's law, it decreases the amount of wall tension (T) at any given level of volume and pressure:

$$
\mathrm{T}=\mathrm{P} \times \mathrm{V} / \mathrm{h}
$$

While deleterious effects of excessive ventricular hypertrophy are conceivable, imaging 
studies have recently shown that concentric RV hypertrophy improves outcome in patients with severe $\mathrm{PH}^{24,25}$.

\section{PHARMACOLOGY OF RV-ARTERIAL COUPLING IN PULMONARY HYPERTENSION}

There are no reported studies on the effects of pharmacological interventions on RV-arterial coupling in patients with severe $\mathrm{PH}$. One has therefore to rely on translations from experimental animal literature.

Catecholamines are in principle not recommended in severe $\mathrm{PH}$ because of their potential to induce pulmonary vasoconstriction, excessive tachycardia and arrhythmia ${ }^{26}$. Moreover, catecholamines have been associated with increased mortality in RV failure ${ }^{27}$. However, the latter effect is probably due to the fact that these drugs are prescribed in the most severely ill patients. Low-dose catecholamines are often used for short periods of time in the intensive care setting to stabilize patients with acute or acute-on chronic RV failure, buying time for fluid management and treatments targeting the pulmonary circulation. In experimental $\mathrm{PH}$ low-dose dobutamine improved RV-arterial coupling by an inotropic effect with a slight decrease in $\mathrm{PVR}^{15,28}$. Low-dose norepinephrine improved RV-arterial coupling through an exclusive positive inotropic effect, which was however less pronounced than with low-dose dobutamine ${ }^{28}$.

Quite different is the idea that $\beta$-blockers might improve RV failure like in LV failure. Heart failure, whether global or limited to the RV is associated with a neuro-humoral activation which helps initially to preserve blood pressure and glomerular filtration, but in the long term further deteriorates cardiovascular function. Experimental animal evidence is contrasted. Acute administration of propranolol reduced RV-arterial coupling through combined negative inotropy and pulmonary vasoconstriction in acute hypoxic $\mathrm{PH}^{15}$. However, chronic administration of bisoprolol improved RV-arterial coupling by an increased contractility in monocrotaline-induced pulmonary hypertension ${ }^{29}$. Chronic administration of carvedilol or metoprolol improved echocardiography of RV function in $\mathrm{PH}$ induced by monocrotaline injection or by administration of the vascular endothelial growth factor-receptor antagonist SU5416 combined with hypoxia ${ }^{30}$. Accordingly randomized controlled trials of low-dose $\beta$-blockers have been initiated in patients with PAH. The results so far have not been encouraging, with no signal of possible benefit and concerns about side effects and decreased exercise capacity and cardiac output ${ }^{31}$.

There has been suggestion that pharmacological interventions targeting the pulmonary circulation in PAH might also have intrinsic beneficial effects on the RV ${ }^{32,33}$. However, the experimental evidence for such direct myocardial effects in intact animal models of $\mathrm{PH}$ is scarce, with some discrepant results about sildenafil. Prostacyclins decreased Emax in proportion to decreased $\mathrm{Ea}^{34-36}$. Sildenafil improved RV-arterial coupling in hypoxia by exclusive pulmonary vasodilating effects ${ }^{37}$. However, sildenafil improved RV-arterial coupling by a positive inotropic effect in monocrotaline-induced $\mathrm{PH}^{38}$. Bosentan had no intrinsic effect on contractility in $\mathrm{PH}$ induced by 3 months of aorta-pulmonary shunting ${ }^{39}$. 


\section{SIMPLIFIED METHODS FOR THE MEASUREMENT OF RV-ARTERIAL COUPLING}

\section{The pressure method}

With the calculation of a Pmax from a RV pressure curve and assuming that mPAP is a satisfactory estimate of RV pressure at the point of maximal elastance, Emax can be estimated by the ratio of the difference (Pmax - mPAP) to SV, and Ea by the ratio of mPAP to $\mathrm{SV}^{40}$ :

$$
\mathrm{Emax} / \mathrm{Ea}=\mathrm{Pmax} / \mathrm{mPAP}-1
$$

The pressure method has been implemented on digitized right heart catheterization recordings of patients with $\mathrm{PH}$ on left heart diseases (PH-LHD) or PAH. Emax/Ea was depressed in patients with combined pre- and post-capillary $\mathrm{PH}$, not in patients with isolated postcapillary or $\mathrm{PAH}$, and was related to surviv$\mathrm{al}^{41}$. In another study on patients referred for $\mathrm{PH}$, Emax/Ea measured by the pressure method did not emerge as an independent predictor of outcome ${ }^{42}$.

\section{The volume method}

The ratio of elastances Emax/Ea presents with a common pressure term, and can thus also be simplified to a ratio of volumes ${ }^{43}$ :

\section{$\mathrm{ESP} / \mathrm{ESV} / \mathrm{ESP} / \mathrm{SV}=\mathrm{SV} / \mathrm{ESV}$}

The main inherent assumption that the endsystolic PV relationship is linear and crosses the origin ${ }^{40}$. Therefore, the ESP/ESV relationship under-estimates Emax ${ }^{40,42}$.
A simple rearrangement of the $\mathrm{EF}$ equation $\mathrm{EF}=\mathrm{SV} / \mathrm{EDV}$ shows that SV/ESV and EF are nonlinearly related ${ }^{44}$ :

$$
\mathrm{SV} / \mathrm{ESV}=\mathrm{EF} /(1-\mathrm{EF})
$$

The nonlinear relationship between EF and SV/ESV is illustrated in figure 2.

The SV/ESV ratio, not EF has been shown to be an independent predictor of outcome in patients referred for $\mathrm{PH}^{42}$. However, this was not confirmed in a study on patients with established PAH, in whom the SV/ESV and EF performed equally, even though a decrease in SV/ESV was more tightly related to poor outcome $^{45}$. Different predictive capability of SV/ESV and EF may be related to their nonlinear relationship, with SV/ESV being more sensitive than $\mathrm{EF}$ to changes in RV function in less advanced disease (Fig. 2).

Right ventricular EF is a potent independent predictor of survival in $\mathrm{PH}$, with a cut-off value determined by the best combination of sensitivity and specificity of $35 \% 46,47$. The rigorously determined cut-off value for SV/ESV is $51 \%{ }^{42}$. A simple re-arrangement of the $\mathrm{EF}$ and SV/ESV equations allows to demonstrate that below these cut-off values, preservation of SV requires an increase in both ESV and EDV (Fig. 3).

It is important to note that PVR can increase considerably without decrease in $\mathrm{RV} \mathrm{EF}^{46}$ and that RV EF can decrease in spite of therapeutic success to decrease in $\mathrm{PVR}^{47}$. In both cases, outcome is predicted by EF, not $\mathrm{PVR}^{44,45}$. A decrease in PVR at increased cardiac output cannot unload the RV if not accompanied by a marked decrease in $\mathrm{PVR}^{48}$. 


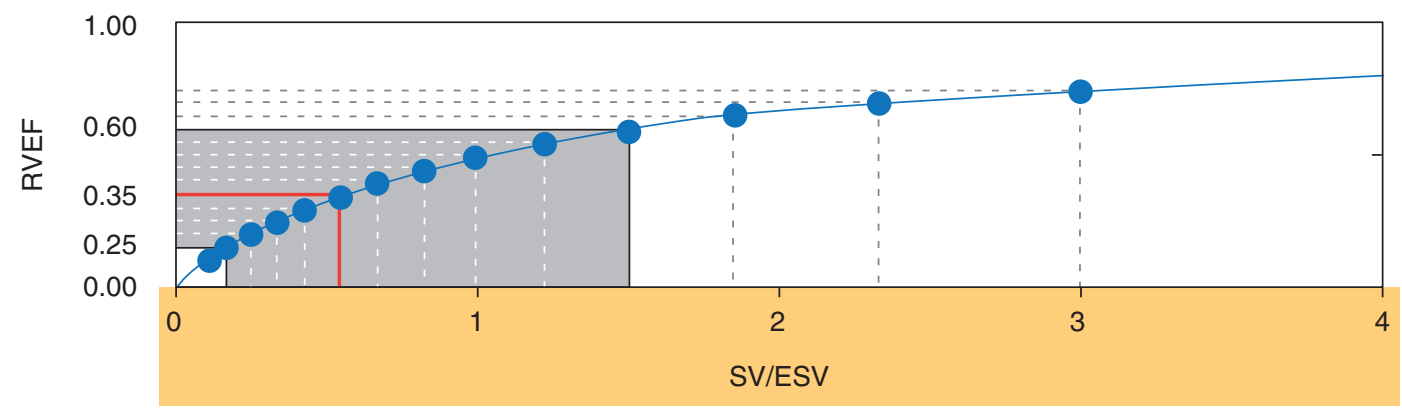

Figure 2. Curvilinear relationship between right ventricular (RV) ejection fraction (EF) and ratio of stroke volume (SV) to end-systolic volume (ESV). The grey region shows the pulmonary hypertension RVEF range (0.15-0.60) and the red line shows the RVEF and corresponding SV/ESV cut-off value that is predictive of outcomes (reproduced with permission from Vanderpool RR et al.44).
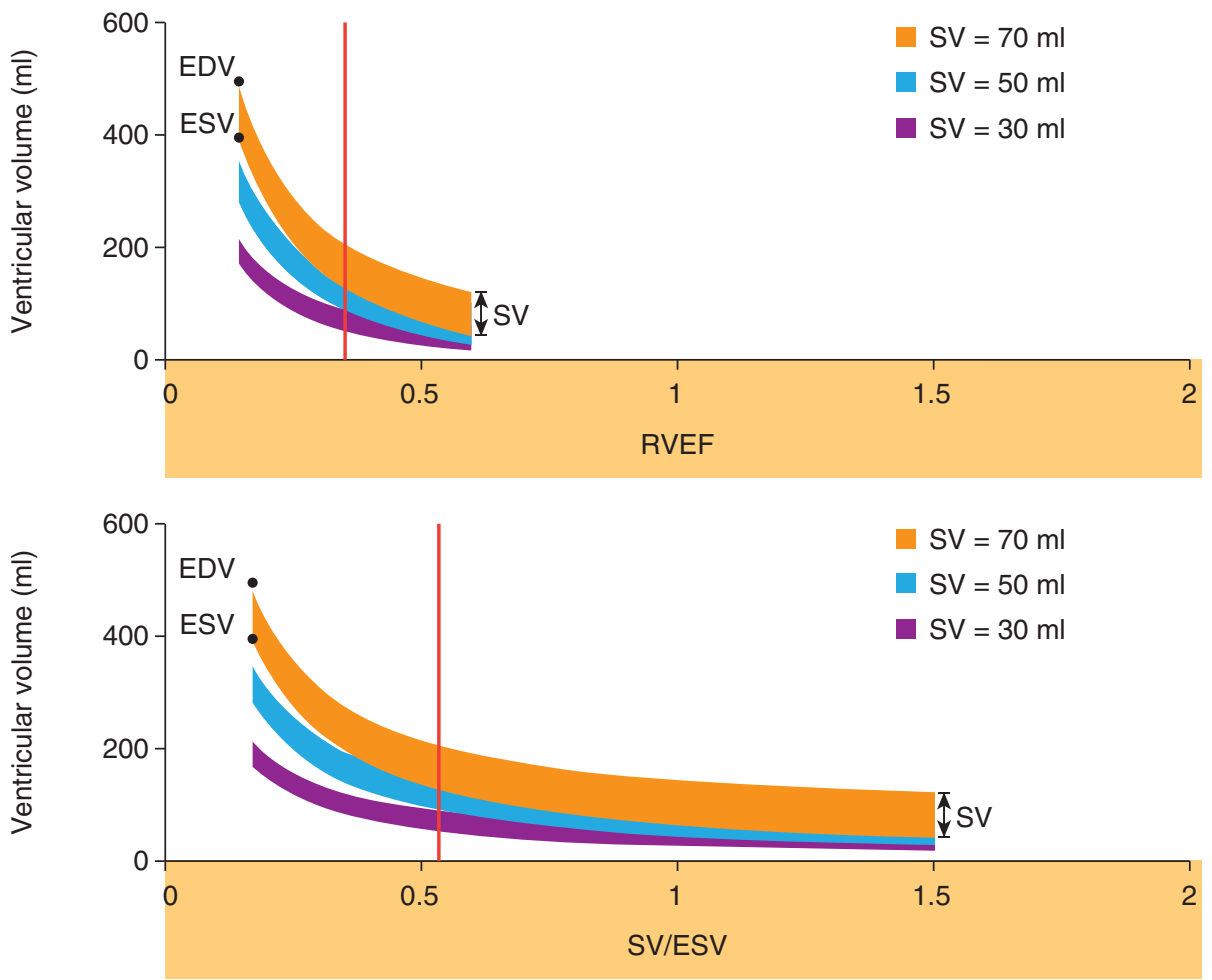

Figure 3. Non-linear relationship between right ventricular (RV) end-diastolic volume (EDV) and end-systolic volume (ESV), and respectively ejection fraction (EF) and ratio of stroke volume (SV) to ESV at a given SV. As RVEF and SV/ESV decrease, ESV and EDV have to increase to maintain SV. The red line shows the RVEF and corresponding SV/ESV cut-off value that has been shown to be predictive of outcomes (reproduced with permission from Vanderpool RR et al. ${ }^{44}$ ). 
A hyper-dynamic state with little effect on PAP, as was sometimes reported with high doses of prostacyclins in $\mathrm{PAH}^{32}$ can only but increase RV afterload, which is not a desirable objective.

Advances in 3-dimensional echocardiography now offer the prospect of easier bedside measurements of RV volumes ${ }^{49}$, and thus of EF or SV/ESV for the evaluation of RV-arterial coupling.

\section{The contractile reserve}

Systolic function adaptation to afterload can also be tested dynamically to determine a contractile reserve, or the capacity to increase contractility at a given level of loading. Contractile or ventricular reserve determined using exercise or pharmacological stress tests (typically an infusion of dobutamine) has been shown to be a strong predictor of outcome in heart failure ${ }^{50}$.

As a simple noninvasive approach to assess RV contractile reserve, Doppler echocardiography was used to measure RV systolic pressure from the maximum velocity of tricuspid regurgitation at rest and at exercise in patients with either PAH or $\mathrm{CTEPH}^{51}$. An exercise-induced increase in systolic RV pressure by more $30 \mathrm{mmHg}$ was a strong predictor of exercise capacity and better survival. It was however subsequently shown that the increase in PAP (or systolic RV pressure) during exercise in severe $\mathrm{PH}$ is but loosely correlated to $\mathrm{Emax} / \mathrm{Ea}^{19}$ so that there is uncertainty as to whether the measurement effectively addresses the RV rather than the pulmonary circulation.
Other tentative assessments of RV contractile reserve have relied on low-dose dobutamine to increase tricuspid annular plane systolic excursion (TAPSE) or tissue Doppler tricuspid annulus $S^{\prime}$ wave ${ }^{52}$ or exercise-induced changes in TAPSE/PAP ratio ${ }^{53}$. While dobutamine-induced increase in indices of RV systolic function are indeed tightly related to resting Emax/ $\mathrm{Ea}^{54}$ the optimal protocol to assess RV contractile reserve remains to be validated.

\section{DIASTOLIC FUNCTION}

Even though systolic function adaptation to afterload is central to RV failure symptomatology, diastolic function (mal)adaptation must also be taken into consideration.

Diastolic function is described by a diastolic elastance curve determined by a family of pressure-volume loops at variable loading. It is curvilinear thus impossible to summarize as a single number. Several formulas fitting two or three diastolic PV coordinates have been proposed ${ }^{8}$. Most recently RV diastolic stiffness was estimated in 21 patients with PAH by fitting a non-linear exponential curve with the formula $\mathrm{P}=\alpha\left(\mathrm{e}^{\mathrm{V} \beta}-1\right)$, where $\alpha$ is a curve fitting constant and $\beta$ a diastolic stiffness constant ${ }^{55}$. These results were confirmed in a larger cohort of 63 patients but with diastolic function simplified as a single end-diastolic PV ratio ${ }^{56}$.

Whether RV diastolic function however defined might be an independent predictor of outcome in $\mathrm{PH}$ is not always confirmed ${ }^{42}$. Another still unsolved issue is whether diastolic function might deteriorate independently of systolic function. 


\section{VENTRICULAR INTERACTION}

RV function cannot be dissociated from LV function. Both structures have the septum and free wall myocardial fibres in common, and are constrained within a non-distensible pericardial envelope. There is thus direct interaction, or ventricular interdependence, defined as the forces that are transmitted from one ventricle to the other ventricle through the myocardium and pericardium, independent of neural, humoral or circulatory effects ${ }^{57}$.

Diastolic ventricular interaction refers to the competition for space within the pericardium when the RV dilates, which alters LV filling and may be a cause of inadequate cardiac output response to metabolic demand. Right heart catheterization and imaging studies have shown that in patients with severe $\mathrm{PH}, \mathrm{mPAP}$ and LV peak filling rate are altered in proportion to decreased RV EF ${ }^{58}$.

Systolic interaction refers to positive interaction between RV and LV contractions. It can be shown experimentally that aortic constriction and associated enhanced LV contraction, markedly improve RV function in animals with pulmonary artery banding ${ }^{59}$. Similarly, in electrically isolated ventricular preparations in the otherwise intact dog heart, LV contraction contributes a significant amount $(\sim 30 \%)$ to both RV contraction and pulmonary flow $^{60}$. This is explained by a mechanical entrainment effect, but also by LV systolic function determining systemic blood pressure, which is an essential determinant of RV coronary perfusion. Increased RV filling pressures and excessive decrease in blood pressure may be a cause of RV ischemia and decreased contractility in severe $\mathrm{PH}^{61}$.
An additional cause of negative ventricular interaction disclosed by magnetic resonance imaging studies is regional and inter-ventricular asynchrony with post-systolic contraction or "shortening", which has been shown to develop in parallel to increased PAP and contributes to altered RV systolic function and LV under-filling ${ }^{62}$. Inter-ventricular asynchrony can be assessed by tissue Doppler imaging ${ }^{63}$. The RV regional inhomogeneity of contraction or dyssynchrony can now accurately be identified and quantified by speckle tracking echocardiography ${ }^{64-66}$. RV dyssynchrony alters systolic function and is associated with a poor survival ${ }^{64-66}$.

In addition to direct interaction, $\mathrm{RV}$ and/or LV failure trigger an activation of the sympathetic nervous and renin-angiotensin-aldosterone systems which may deteriorate function of the initially unaffected ventricle and contribute to worsen symptomatology, functional state and outcome.

\section{RESPIRATORY SYSTEM MECHANICS}

High or low lung volumes increase PVR ${ }^{67}$. This is caused by dominant effects of compression of alveolar vessels at high lung volumes and a compression of extra-alveolar vessels at low lung volumes. PVR is minimal at functional residual capacity.

Breathing is necessarily associated with pleural pressure $(\mathrm{Ppl})$ changes. The amplitude of $\mathrm{Ppl}$ variations is proportional to tidal volume and respiratory system compliance, and tends to be on average positive during mechanical ventilation and negative during spontaneous breathing. The heart is exposed a surrounding 
pressure approximately equal to Ppl, which is on average 3-4 mmHg below atmospheric pressure. Respiratory Ppl swings do not affect trans-mural RV, pulmonary vascular and LV pressures. However, LV-arterial coupling is affected as the aorta is in open communication with the systemic arterial system. Positive intra-thoracic pressure decreases the pressure gradient needed to open the aortic valve, and thereby decreases LV afterload, in addition to a preload reduction due to a decrease in systemic venous return ${ }^{68}$. This carries a risk of insufficient preload adaptation to afterload in advanced PH and RV failure. Negative intra-thoracic pressure increases the pressure gradient to open the aortic valve, and thus increases LV afterload - while systemic venous return is slightly increased or maintained ${ }^{69}$. Markedly negative intra-thoracic pressure may be a cause of left heart failure on weaning from mechanical ventilation ${ }^{70}$. Negative Ppl with hyper-inflation in spontaneously breathing patients may thus cause worsening $\mathrm{PH}$ on upstream transmission of pulmonary artery wedge pressure (PAWP).

An additional effect of disturbed respiratory system mechanics is due to dynamic hyper-inflation in patients with obstructed airways ${ }^{71}$. This causes prolonged positive Ppl during expiration and is a cause of over-estimation of PAP and PAWP by reading pulmonary vascular pressure curves at end-expiration.

In the presence of excessive intra-thoracic pressure swings, with or without dynamic hyper-inflation, the reading of pulmonary vascular pressures has to be corrected for Ppl, or, as an acceptable approximation, averaged over several respiratory cycles ${ }^{72}$. This notion was recently incorporated in the European guidelines ${ }^{1}$.
Lung disease can also is of course a cause of hypoxic vasoconstriction which, if globally distributed over the lungs, may aggravate $\mathrm{PH}^{73}$.

\section{A GLOBAL VIEW ON RV FAILURE}

Thus, PH increases RV afterload requiring a homeometric adaptation with eventual hypertrophy. When this adaptation fails, the RV enlarges, decreasing LV preloading because of competition for space within the pericardium. This decreases SV and blood pressure, with negative systolic interaction as a cause of further RV-arterial uncoupling, which may be aggravated by RV ischemia from decreased coronary perfusion pressure (gradient between diastolic blood pressure and right atrial pressure).

These interactions may allow one to identify targets of interventions. The first target is increased PVR, which can be controlled by thrombolytics in severe pulmonary embolism, inhaled NO, inhaled iloprost in case of acute pulmonary vasoconstriction, prostacyclins, intravenous epoprostenol, PDE5i's and ERA's for PAH, correction of decreased or increased functional residual capacity, $\mathrm{pH}$ and hypoxemia all causes of increased PVR in lung diseases. The second target is RV contractility, which can be temporarily improved by intravenous dobutamine. The third target is excessive RV preload and associated negative diastolic interaction which can be controlled by high-dose intravenous diuretics and fluid restriction, or hemo-filtration in case of renal insufficiency. The fourth target is hypotension and associated negative systolic interaction and eventual RV ischemia, which can be controlled by intravenous norepinephrine. 


\section{CONCLUSIONS}

\section{The interactions between the heart and the normal or hypertensive pulmonary Circula- tion. are complex. Their understanding offers indispensable guidance to differential diag- nosis and therapeutic options.}

\section{CONFLICT OF INTEREST}

\section{Dr. Robert Naeije has nothing to disclose.}

\section{REFERENCES}

1. Galiè N, Humbert M, Vachiery JL et al. 2015 ESC/ERS Guidelines for the diagnosis and treatment of pulmonary hypertension: The Joint Task Force for the Diagnosis and Treatment of Pulmonary Hypertension of the European Society of Cardiology (ESC) and the European Respiratory Society (ERS) Endorsed by: Association for European Paediatric and Congenital Cardiology (AEPC), International Society for Heart and Lung Transplantation (ISHLT). Eur Heart J. 2016;37:67-119.

2. Vonk-Noordegraaf A, Haddad F, Chin KM et al. Right heart adaptation to pulmonary arterial hypertension: physiology and pathobiology J Am Coll Cardiol. 2013;62(25 Suppl):D22-33.

3. Haddad F, Hunt SA, Rosenthal DN, Murphy DJ. Right ventricular function in cardiovascular disease, Part I. Anatomy, physiology, aging and functional assessment of the right ventricle. Circulation. 2008;117:1436-48.

4. Patterson SW, Piper H, Starling EH. The regulation of the heart beat. J Physiol. 1914;48:465-513.

5. Anrep von G. On the part played by the suprarenals in the normal vascular reactions of the body. J Physiol. 1912;45:307-17.

6. Naeije R, Manes A. The right ventricle in pulmonary arterial hypertension. Eur Respir Rev. 2014;23:476-87.

7. Gewillig M. The Fontan circulation. Heart. 2005;91:839-46.

8. Vonk-Noordegraaf A, Westerhof N. Describing right ventricular function. Eur Respir J. 2013;41:1419-23.

9. Saouti N, Westerhof N, Helderman F et al. Right ventricular oscillatory power is a constant fraction of total power irrespective of pulmonary artery pressure. Am J Respir Crit Care Med. 2010;182:1315-20.

10. Suga H, Sagawa K, Shoukas AA. Load independence of the instantaneous pressure-volume ratio of the canine left ventricle and effects of epinephrine and heart rate on the ratio. Circ Res. 1973;32:314-22.

11. Maughan WL. Shoukas AA, Sagawa K, Weisfeldt ML. Instantaneous pressure-volume relationship of the canine right ventricle. Circ Res. 1979;44:309-315.

12. Redington AN, Rigby RL, Shinebourne EA, Oldershaw PJ. Changes in pressure-volume relation of the right ventricle when its loading conditions are modified. Br Heart J. 1990;63:45-9.

13. Tedford RJ, Mudd JO, Girgis RE et al. Right ventricular dysfunction in systemic sclerosis associated pulmonary arterial hypertension. Circ Heart Fail. 2013;6:953-63.

14. Sunagawa K, Yamada A, Senda Y, Kikuchi Y, Nakamura M, Shibahara T. Estimation of the hydromotive source pressure from ejecting beats of the left ventricle. IEEE Trans Biomed Eng. 1980;57:299-305.
15. Brimioulle S, Wauthy P, Ewalenko P et al. Single-beat estimation of right ventricular end-systolic pressure-volume relationship. Am J Physiol Heart Circ Physiol. 2003;284:H1625-H1630.

16. Dell'Italia LJ,Walsh RA. Application of a time-varying elastance model to right ventricular performance in man. Cardiovasc Res. 1988;22:864-74.

17. Kuehne T, Yilmaz S, Steendijk P et al. Magnetic resonance imaging analysis of right ventricular pressure-volume loops: in vivo validation and clinical application in patients with pulmonary hypertension. Circulation. 2004;110:2010-6.

18. McCabe C, White PA, Hoole SP et al. Right Ventricular dysfunction in chronic thromboembolic obstruction of the pulmonary artery. J Appl Physiol. 2013;116:355-63

19. Spruijt OA, de Man FS, Groepenhoff $\mathrm{H}$ et al. The effects of exercise on right ventricular contractility and right ventricular-arterial coupling in pulmonary hypertension. Am J Respir Crit Care Med. 2015;191:1050-7.

20. Hsu S, Houston BA, Tampakakis E et al. Right Ventricular Functional Reserve in Pulmonary Arterial Hypertension. Circulation. 2016;133:2413-22.

21. Wauthy P, Naeije R, Brimioulle S. Left and right ventriculo-arterial coupling in a patient with congenitally corrected transposition. Cardiol Young. 2005; 15:647-9.

22. Rosenblueth A, Alanis J, Lopez E, Rubio R. The adaptation of ventricular muscle to different circulatory conditions. Arch Int Physiol Biochim. 1959 67:358-73.

23. van de Veerdonk MC, Marcus JT, Westerhof N et al. Signs of right ventric ular deterioration in clinically stable patients with pulmonary arterial hypertension. Chest. 2015;147:1063-71.

24. Badagliacca R, Poscia R, Pezzuto B et al. Right ventricular remodeling in idiopathic pulmonary arterial hypertension: adaptive versus maladaptive morphology. J Heart Lung Transplant. 2015;34:395-403.

25. Badagliacca R, Poscia R, Pezzuto B et al. Right ventricular concentric hypertrophy and clinical worsening in idiopathic pulmonary arterial hypertension. J Heart Lung Transplant. 2016;35:1321-9.

26. Hoeper MM, Granton J. Intensive care unit management of patients with severe pulmonary hypertension and right heart failure Am J Respir Crit Care Med. 2011;184: 1114-24.

27. Sztrymf B, Günther S, Artaud-Macari E et al. Left ventricular ejection time in acute heart failure complicating pre-capillary pulmonary hypertension. Chest. 2013;144:1512-20.

28. Kerbaul F, Rondelet B, Motte S et al. Effects of norepinephrine and dobutamine on pressure load-induced right ventricular failure. Crit Care Med. 2004;32:1035-40.

29. de Man FS, Handoko ML, van Ballegoij JJ et al. Bisoprolol delays progression towards right heart failure in experimental pulmonary hypertension. Circ Heart Fail. 2012;5:97-105.

30. Bogaard HJ, Natarajan R, Mizuno S et al. Adrenergic blockade reverses right heart remodeling and dysfunction in pulmonary hypertensive rats. Am J Respir Crit Care Med. 2010;182:652-6.

31. van Campen JS, de Boer K, van de Veerdonk MC et al. Bisoprolol in idiopathic pulmonary arterial hypertension: an explorative study. Eur Respir J. 2016;48:787-96

32. Rich S, McLaughlin VV. The effects of chronic prostacyclin therapy on cardiac output and symptoms in primary pulmonary hypertension. J Am Coll Cardiol. 1999;34:1184-7.

33. Nagendran J, Archer SL, Soliman D et al. Phosphodiesterase type 5 is highly expressed in the hypertrophied human right ventricle, and acute inhibition of phosphodiesterase type 5 improves contractility. Circulation. 2007;116:238-248.

34. Wauthy P, Kafi AS, Mooi W, Naeije R, Brimioulle S. Effects of nitric oxide and prostacyclin in an over-Circulation. model of pulmonary hypertension J Thorac Cardiovasc Surg. 2003;125:1430-7.

35. Kerbaul F, Brimioulle S, Rondelet B, Dewachter C, Hubloue I, Naeije R. How prostacyclin improves cardiac output in right heart failure in conjunction with pulmonary hypertension. Am J Respir Crit Care Med. 2007;175:846-50.

36. Rex S, Missant C, Segers P, Rossaint R, Wouters PF. Epoprostenol treatment of acute pulmonary hypertension is associated with a paradoxical decrease in right ventricular contractility. Intens Care Med. 2008;34:179-89. 
37. Fesler P, Pagnamenta A, Rondelet B, Kerbaul F, Naeije R. Effects of sildenafil on hypoxic pulmonary vascular function in dogs. J Appl Physiol 2006;101:1085-1090.

38. Borgdorff MA, Bartelds B, Dickinson MG et al. Sildenafil enhances systolic adaptation, but does not prevent diastolic dysfunction, in the pressure-loaded right ventricle. Eur J Heart Fail. 2012;14:1067-74.

39. Rondelet B, Kerbaul F, Motte S et al. Bosentan for the prevention of overcirculation-induced pulmonary hypertension. Circulation. 2003;107: 1329-35.

40. Trip P, Kind T, van de Veerdonk MC et al. Accurate assessment of load-independent right ventricular systolic function in patients with pulmonary hypertension. J Heart Lung Transplant. 2013;32:50-5.

41. Gerges M, Gerges C, Pistritto AM et al. Pulmonary hypertension in heart failure: epidemiology, right ventricular function and survival. Am J Respir Crit Care Med. 2015;192:1234-46.

42. Vanderpool RR, Pinsky MR, Naeije R et al. Right ventricular-pulmonary arterial coupling predicts outcome in patients referred for pulmonary hypertension. Heart. 2015;101:37-43.

43. Sanz J, García-Alvarez A, Fernández-Friera L et al. Right ventriculo-arterial coupling in pulmonary hypertension: a magnetic resonance study. Heart. 2012;98:238-43.

44. Vanderpool RR, Rischard F, Naeije R, Hunter K, Simon MA. Simple functional imaging of the right ventricle in pulmonary hypertension: Can right ventricular ejection fraction be improved? Int J Cardiol. 2016;223:93-4.

45. Brewis MJ, Bellofiore A, Vanderpool RR et al. Imaging right ventricular function to predict outcome in pulmonary arterial hypertension. Int J Cardiol. 2016;218:206-11.

46. Ghio S, Gavazzi A, Campana C et al. Independent and additive prognostic value of right ventricular systolic function and pulmonary artery pressure in patients with chronic heart failure. J Am Coll Cardiol. 2001;37:183-8.

47. van de Veerdonk MC, Kind T et al. Progressive right ventricular dysfunction in patients with pulmonary arterial hypertension responding to therapy. J Am Coll Cardiol. 2011;58:2511-9.

48. Sniderman AD, Fitchett DH. Vasodilators and pulmonary arterial hypertension: the paradox of therapeutic success and clinical failure. Int J Cardiol. 1988;20:173-81.

49. Zhang QB, Sun JP, Gao RF et al. Feasibility of single-beat full volume capture real-time three-dimensional echocardiography for quantification of right ventricular volume: validation by cardiac magnetic resonance imaging. Int J Cardiol. 2013;168: 3991-5.

50. Haddad F, Vrtovec B, Ashley EA, Deschamps A, Haddad H, Denault AY. The concept of ventricular reserve in heart failure and pulmonary hypertension: an old metric that brings us one step closer in our quest for prediction. Curr Opin Cardiol. 2011;26:123-31.

51. Grünig E, Tiede H, Enyimayew EO et al. Assessment and prognostic relevance of right ventricular contractile reserve in patients with pulmonary arterial hypertension. Circulation. 2013;128:2005-15.

52. Sharma T, Lau EM, Choudhary P et al. Dobutamine stress for evaluation of right ventricular reserve in pulmonary arterial hypertension. Eur Respir J. 2015;45:700-8.

53. Guazzi M, Villani S, Generati G et al. Right ventricular contractile reserve and pulmonary Circulation. uncoupling during exercise challenge in heart failure: pathophysiology and clinical phenotypes. JACC Heart Fail. 2016; 4:625-35.

54. Guihaire J, Haddad F, Noly PE et al. Right ventricular reserve in a piglet model of chronic pulmonary hypertension. Eur Respir J. 2015;45:709-17.
55. Rain S, Handoko ML, Trip P et al. Right ventricular diastolic impairment in patients with pulmonary arterial hypertension. Circulation. 2013;128:2016-25.

56. Trip P, Rain S, Handoko ML, van der Bruggen C et al. Clinical relevance of right ventricular diastolic stiffness in pulmonary hypertension. Eur Respir J. 2015;45:1603-12.

57. Santamore WP, Dell'Italia LJ Ventricular interdependence: significant left ventricular contributions to right ventricular systolic function Progr Cardiovasc Dis 1998;40:289-308.

58. Lazar JM, Flores AR, Grandis DJ, Orie JE, Schulman DS. Effects of chronic right ventricular pressure overload on left ventricular diastolic function. Am J Cardiol. 1993;72:1179-82.

59. Belenkie I, Horne SG, Dani R, Smith ER, Tyberg JV. Effects of aortic constriction during experimental acute right ventricular pressure loading. Further insights into diastolic and systolic ventricular interaction. Circulation. 1995;92:546-54.

60. Damiano RJ Jr, La Follette P Jr, Cox JL, Lowe JE, Santamore WP. Significant left ventricular contribution to right ventricular systolic function. Am J Physiol. 1991;261(5 Pt 2):H1514-24.

61. Gómez A, Bialostozky D, Zajarias A et al. Right ventricular ischemia in patients with primary pulmonary hypertension. J Am Coll Cardiol. 2001;381137-41.

62. Marcus JT, Gan CT, Zwanenburg JJ et al. Interventricular mechanical asynchrony in pulmonary arterial hypertension: left-to-right delay in peak shortening is related to right ventricular overload and left ventricular underfilling. J Am Coll Cardiol. 2008; 51:750-7.

63. Huez S, Faoro V, Vachiery JL, Unger P, Martinot JB, Naeije R. Images in cardiovascular medicine. High-altitude-induced right-heart failure. Circulation. 2007;115:e308-9.

64. Badagliacca R, Reali M, Poscia R et al. Right intraventricular dyssynchrony in idiopathic, heritable, and anorexigen-induced pulmonary arterial hypertension: clinical impact and reversibility. JACC Cardiovasc Imaging. 2015;6: 642-52.

65. Badagliacca R, Poscia R, Pezzuto B et al. Right ventricular dyssynchrony in idiopathic pulmonary arterial hypertension: determinants and impact on pump function. J Heart Lung Transplant. 2015;34:381-9.

66. Smith BC, Dobson G, Dawson D, Charalampopoulos A, Grapsa J, Nihoyannopoulos P._Three-dimensional speckle tracking of the right ventricle: toward optimal quantification of right ventricular dysfunction in pulmonary hypertension. J Am Coll Cardiol. 2014;64:41-51.

67. Wittenberger JL, McGregor M, Berglund E, Borst HG. Influence of state of inflation of the lung on pulmonary vascular resistance. J Appl Physiol. 1960;15:878-82.

68. Scharf SM. Cardiovascular effects of positive pressure ventilation. J Crit Care. 1992;7:268-79.

69. Buda AJ, Pinsky MR, Ingels NB et al. Effects of intrathoracic pressure on left ventricular performance. N Engl J Med. 1979;301:453-9.

70. Lemaire F, Teboul JC, Cinotti L et al. Acute left ventricular dysfunction during unsuccessful weaning from mechanical ventilation. Anesthesiology. 1988;69:171-6.

71. Pepe PE, Marini JJ. Occult positive end-expiratory pressure in mechanically ventilated patients with airflow obstruction (the auto-PEEP effect) Am Rev Respir Dis. 1982;126:166-70.

72. Kovacs G, Avian A, Pienn M, Naeije R, Olschewski H. Reading pulmonary vascular pressure tracings. How to handle the problems of zero leveling and respiratory swings. Am J Respir Crit Care Med. 2014;190:252-7.

73. Hughes JMB. Hypoxic pulmonary vasoconstriction: clinical implications. Eur Respir J. 2016;47:31-4. 